\title{
Notas sobre el realismo selectivo y sus respuestas a los problemas del realismo científico*
}

\author{
Notes on selective realism and its answer to the problem of scientific realism \\ BENEDICTO ACOSTA ** \\ Universidad de Salamanca, bnecosta@usal.es \\ Recibido el 28 de noviembre de 2020, APRobado el 17 de diciembre de 2020
}

\begin{abstract}
Resumen
El objetivo es presentar brevemente tres corrientes del realismo selectivo en su relación con el realismo científico en general, a saber, el realismo estructural, el explicacionismo y el realismo de entidades. Primeramente, se define el realismo filosófico y se caracterizan el realismo científico y el realismo selectivo. Se parte de la hipótesis de que la principal virtud del selectivismo es poder responder al problema de las entidades inobservables en ciencia y al problema de la metainducción histórica de las teorías, algo que las conecta con otras propuestas realistas. Se corrobora esta hipótesis en los apartados dedicados a estas corrientes, que poseen una misma estructura: primero se expone la respuesta que dan al problema de las entidades, seguida por la respuesta al problema teórico que implica el argumento de la metainducción. Tras todo ello, se presentan también algunas críticas abiertas a cada corriente.
\end{abstract}

\section{Palabras Clave}

Realismo científico, selectivismo, inobservables, metainducción.

\begin{abstract}
The aim of this paper is to briefly present three streams of selective realism, namely structural realism, explanationism, and entity realism, and their relation to scientific realism in general. Firstly, we define philosophical realism; then, scientific realism and selective realism are both characterized. The hypothesis is that the main virtue of selectivism is being able to respond to the problem of unobservable entities in science and to the problem of the historical meta-induction of theories, something that connects it with other realistic proposals. This hypothesis is corroborated in the sections dedicated to these currents, which have the same structure: first, the answer they give to the problem of entities is exposed, followed by the answer to the theoretical problem that the meta-induction argument implies. After all this, there are also some open criticisms of each current.
\end{abstract}

\section{KeYwORDS}

Scientific realism, selectivism, unobservables, meta-induction.

\footnotetext{
*“Esta investigación ha sido posible gracias a un contrato de Formación del Profesorado Universitario (FPU19/03734), financiado por el Ministerio de Universidades español".

** (1) orcid.org/0000-0003-0878-0657 Google Scholar
} 


\section{Realismo, realismo científico y realismos selectivos}

El realismo filosófico acoge a una serie amplia de doctrinas. "Realismo" es una palabra que suele venir acompañada de adjetivos: realismo ontológico, realismo epistemológico, incluso Nuevo realismo o Realismo Crítico. Lo que todos parecen compartir es la defensa de que una determinada realidad natural (por ejemplo, un átomo, un cangrejo, aunque no un Ministerio de Hacienda ${ }^{1}$ ) es independiente de nosotros, es decir, de la relación que mantenemos con ella; y que, en el mejor de los casos, esta puede ser una realidad cognoscible.

En un reciente diálogo entre Bas van Fraassen y Anjan Chakravartty, el primero de ellos comentaba sobre el realismo científico lo siguiente: "[el realista científico] es alguien que sostiene [...] que las entidades no-observables existen" (12. La traducción es mía). Siempre que estos objetos inobservables sean parte del mundo natural, en ningún sentido ello contraviene la primera definición. Estos objetos existen, pues, con independencia de nosotros, aunque no podamos afirmar que nuestras representaciones sean fieles a dicha existencia.

Resulta también fácil de entender que el científico profesional se encuentre, en su práctica diaria, con conceptos abstractos de ese tipo, y que su respuesta consista en creer que dichas entidades gozan de cierta "autonomía", en el sentido de que la inquisición sobre su naturaleza o conducta no las invente, no las haga reales ni las introduzca en el mundo. Es una actitud en cierto sentido "natural", o nada descabellada, y concordante con las intuiciones más habituales que tenemos los seres humanos. Chakravartty coincide con su compañero al interpretar a los realistas de este modo, pero hace bien en resaltar el hecho de que por esta línea argumental el realista queda reducido a alguien que necesitaría comprometerse demasiado con la existencia de los modelos científicos, cuando no de las idealizaciones (como cuando se habla un gas ideal).

¿Cuál es, pues, la realidad en que el realista científico cree? La pregunta es crucial, aunque se responde de un modo diferente según se atienda a las entidades o a las teorías. Al final del escrito, ninguno de los dos filósofos llega a una conclusión, aunque Chakravartty termina por afirmar que tanto en el caso de que se afirme la realidad del lenguaje

\footnotetext{
${ }^{1}$ Esta distinción entre realidad natural y realidad social la tomo de Ferraris. Manifiesto del Nuevo Realismo. Ariadna ediciones. 2012.
} 
teórico, como si se sostiene respecto de las entidades inobservables, lo importante para el realista es que se comporte como si algunas de ellas existiesen. También lo señala en este otro escrito: "El realismo científico es esa actitud epistémica positiva respecto al contenido de nuestras mejores teorías y modelos, y recomienda creer tanto en los aspectos observables como los no-observables del mundo descrito por las ciencias" (Chakravarrty 1. La traducción es mía).

Entre los realismos científicos hay proyectos que han dado en llamarse "selectivistas". La hipótesis de este trabajo es que comparten la creencia de que ellos pueden enfrentar de un mejor modo que el realismo científico tradicional:

(1) el problema de los objetos inobservables, al que ya se ha hecho referencia.

(2) Y, sobre todo, el argumento de la meta-inducción histórica pesimista, que pone en seria duda la verdad de nuestras teorías. Este argumento (Laudan, "A convergent") puede reformularse como sigue: el hecho de haber descubierto o revelado la falsedad de las teorías pasadas -incluso de aquellas teorías que tuvieron éxito empírico- nos lleva a pensar que las teorías presentes también están erradas.

Chakravarty distingue tres tipos de selectivismos (Chakravartty cap. 2.3): el realismo estructural, el realismo deentidades " explicacionismo" (explanationism). Contrastan entre sí en el problema principal que intentan enfocar. Mientras que el realismo de entidades se compromete con la existencia de algunos inobservables, el "explicacionismo" también procura dar respuesta al argumento de la meta-inducción. Alberto Cordero, defensor del realismo selectivo, piensa que "es en principio posible tener razones de peso para suponer que algunas teorías son aproximadamente más ciertas que otras" (Cordero, Sobre el mito 364), algo que muchos realistas selectivos repiten.

Autores como Cordero se comprometen con un realismo de tipo selectivo porque se refieren a "porciones significativas" de nuestros asertos teóricos, y no piensan en "teorías completamente verdaderas" o "teorías verosímiles". El principal problema al que a su vez se enfrenta el proyecto selectivista es la imputación, por parte de los realistas más ortodoxos, del olvido de los fundamentos compartidos, del núcleo auténticamente realista. Por mor de la concisión, no se expondrán estas 
críticas. Se procurará simplemente contraponer las respuestas que algunas corrientes selectivistas dan al problema de los inobservables y al problema de la falsedad de las teorías empíricamente exitosas, que son problemas clásicos del realismo científico.

La posición selectivista respecto a las teorías coincide, pues, en el que el éxito no es algo que se predique de la teoría in toto. Dicho con otras palabras: las teorías falsas puedan contener enunciados verdaderos. Lo que sigue de este artículo se divide en tres apartados, cada uno de ellos dedicado a una de estas corrientes; apartados que poseen una misma estructura: primero se expone la respuesta que dan al problema de las entidades, seguida por la respuesta al problema teórico que implica el argumento de la meta-inducción. Tras todo ello, se presentan también algunas críticas abiertas a cada corriente.

\section{Realismo estructural}

La cualidad veritativa de las teorías es un asunto focal en el selectivismo. Un pariente cercano del explicacionismo de filósofos como Cordero es el llamado "realismo estructural". La actitud epistémica es muy similar, aunque los resultados son distintos. Comencemos por esbozar aquí sus planteamientos fundamentales.

El realismo estructural es a veces llamado también "semántico", y postula que en los saltos de una teoría a otra hay determinadas formas o estructuras que suelen mantenerse. Por ejemplo, entre las teorías sobre la gravitación de Newton a Einstein o en el paso de Fresnel a Maxwell. Estas estructuras suelen ser matemáticas. John Worrall, padre del realismo estructural, dice que, desde el punto de vista de Maxwell, la teoría de Fresnel había malinterpretado la naturaleza de la luz. Sin embargo, si se atiende a la estructura que Fresnel propuso para la luz, más allá de las supuestas oscilaciones del éter, se observa que ésta obedecía a leyes aplicables a un medio mecánico. "Aunque Fresnel estaba equivocado sobre aquello que oscilaba, estaba en lo cierto no solo sobre el fenómeno óptico, sino sobre su dependencia de las ciertas oscilaciones en ángulo recto con la luz" (Worrall 118).

Si se puede demostrar que las mismas ecuaciones son parte de teorías diferentes, cabe pensar que ellas sean las que representen el mundo. Pero como tampoco es necesario preguntarse por lo que esta elaboración matemática denota, o por aquello a lo que refiere (que pueden ser 
entidades inobservables, como un campo magnético), la afirmación de que estas estructuras están asidas, aferradas, al mundo, es hipotética, conjetural. Destaca la famosa sentencia de John Worrall, según la cual el realismo estructural (entendido como un tipo de realismo selectivo) mantiene "lo mejor de los dos mundos": el argumento realista de que las teorías no son exitosas por fruto de la casualidad, y el contrargumento según el cual la demostración como falsas de teorías pasadas exitosas alienta la duda sobre la verdad de las mejores teorías actuales.

La posición del realismo estructural sobre los inobservables no es difícil de intuir a partir de lo que hemos dicho. Huelga señalar que existen numerosas especies dentro del género del realismo estructural (óntico eliminativista, epistémico...) aunque, por lo general, todas afirman que las teorías no describirían adecuadamente la naturaleza de las entidades inobservables, pero sí ciertas relaciones entre ellas (Chakravartty cap. 2.3). Si se observa con detenimiento podrá verse que el compromiso de Worrall está orientado, como aparece en el anterior ejemplo de la luz, hacia los términos teóricos, aunque en una defensa que hace compatible la estructura matemática "con ontologías de individuos" (Madrid-Casado 202).

En principio, a este autor se le sitúa entre los exponentes del realismo estructural epistémico. El propio Worrall, recordemos, hablaba de determinadas "ecuaciones matemáticas" persistentes en el cambio entre teorías, que expresaban "relaciones entre los fenómenos" (122). No obstante, no hacía explícito el modo en que las ecuaciones presentan o expresan la estructura del mundo inobservable. Para Ladyman, quien sostiene un realismo estructural metafísico, las relaciones que dichas ecuaciones reflejan deben ser entendidas como meramente matemáticas, y no tanto como un desvelamiento de entidades inobservables de base.

Una prueba en favor de esta versión del realismo estructural (Rivadulla 23-34) se encuentra en el caso del isomorfismo entre la mecánica matricial y la mecánica ondulatoria, en el seno de la mecánica cuántica. Como explica Madrid-Casado, von Neumann reconstruyó en 1932 ambas teorías como cálculos de operadores sobre espacios de Hilbert, y consiguió concluir que ambas eran matemáticamente equivalentes (puesto que, por decirlo brevemente, los espacios de Hilbert resultaban isomorfos). Así se conseguía explicar la predicción empírica equivalente de ambas mecánicas. Ahora bien, para que dicha identidad en la estructura matemática lo fuera también en el plano 
ontológico, y a partir de ahí nos fuese legítimo inferir algo sobre las entidades inobservables, ambas mecánicas debían poseer la misma cardinalidad, esto es, el mismo número de elementos. Pero no era el caso. Una mecánica proyectaba sobre el mundo una estructura discreta, "y acentuaba una visión corpuscular de los átomos" (Madrid-Casado 204-205), y la otra una estructura continua, centrada en la naturaleza ondulatoria de la cuántica.

Así pues, pareciera que la tendencia está en aclarar a Worrall y evitar compromisos excesivos con ontologías de individuos. Empero, eso puede hacer perder virtualidad a la propuesta del realismo estructural como un selectivismo, al menos en lo que concierne al problema de los inobservables.

\section{Explicacionismo}

Los autores explicacionistas como Stathis Psillos o, sui generis, Alberto Cordero, inciden también en el ejemplo de Fresnel. Los casos que ejemplifican el selectivismo son, como suele ocurrir en toda la filosofía de la ciencia, de la física. Cordero comparte plenamente el hecho descrito por Worrall, a saber, que "en un altísimo grado de aproximación [lo que llamamos luz] se ciñe a las leyes fresnelianas de la reflexión y refracción" (Cordero, Sobre el mito 366). Así pues, la actitud es muy parecida al realismo estructural, puesto que ambos creen que en los cambios de teorías hay algo que se perpetúa; pero las conclusiones son distintas. El explicacionismo nos habla de la existencia de los inobservables de nuestras teorías más exitosas si son relevantes para explicar dicho éxito.

Para Cordero y los explicacionistas, las estructuras matemáticas no son necesariamente lo que se mantiene, porque las "entidades, procesos e historias naturales" también pueden preservarse (Cordero, Mitos y falsedades 18). En cualquier caso, lo que parece imprescindible es que sigan mostrando éxito empírico. Parte de la respuesta que Psillos -quizá el autor más relevante de la corriente- dedica a Laudan tiene que ver con una depuración de la noción de "éxito empírico", tal que pueda revelarse la inexactitud con que Laudan considera como exitosas ciertas teorías pasadas hoy demostradas falsas (Psillos 100110), como la teoría del efluvio. Para Psillos, "una teoría es exitosa syss, permite hacer predicciones novedosas que sean, en principio, testables. En esta dirección, una predicción es novedosa syss, predice 
un fenómeno cuya existencia, está determinada solamente después que la teoría sugiere su existencia, i.e., si el fenómeno era hasta entonces desconocido" (110).

Así pues, reducir el número de ejemplos de que se sirve Laudan para anunciar su argumento es una de las estrategias más habituales, aunque el propio Psillos reconoce que no es suficiente. La doctrina explicacionista respecto a las teorías en general, prefiere negar el carácter pesimista del argumento de la meta-inducción, pues rechaza que suponga una enmienda a la totalidad del realismo. Suelen considerar más bien lo contrario: sorprendentemente, la inducción histórica apoyaría las tesis realistas. Este argumento lo podemos encontrar desarrollado en Psillos (cap. 4 y 5) y, según lo ha reformulado Mauricio Suárez, quien lo denomina "estrategia del argumento de la familiaridad", diría algo así:
... pesar de la falta de referencia de algunos de sus términos [de las teorías pasadas que fueron falsas], las teorías predictivamente exitosas a lo largo de la historia contienen enunciados que nos resultan familiares hoy en día. Tales enunciados, lejos de haber sido rebatidos por la evidencia y, por ende, abandonados a lo largo de la evolución histórica de la ciencia, resultan, en general, aceptados en teorías posteriores. (Suárez 141)

Por ejemplo, aunque el éter luminífero - por seguir con el ejemplose haya convertido hoy en un concepto "extraño" y despojado de cualquier referencia, formaba parte de enunciados en los que también estaban palabras como "ondas", "interferencia" o "luz", que nos siguen resultando "familiares". En general, esta otra estrategia incide en que no se ha podido descubrir la falsedad de las teorías pretéritas básicamente porque la falsedad no es una propiedad de la teoría en su conjunto, sino de determinados enunciados.

Ahora bien, acierta Diéguez al señalar que esto exige un trabajo de criba histórica que demostrase que estas partes aproximadamente verdaderas han sido las causantes o las responsables del éxito de la teoría, "y hacer esto sin que se convierta en una justificación a posteriori de aquello que el realista considera que hay que salvar, es decir, sin preseleccionar estas partes a partir del conocimiento que actualmente tenemos de que, en efecto, fueron las partes que se conservaron de una u otra manera en las teorías posteriores" (Diéguez cap. 3). 
Por ahora, ha quedado demostrado el compromiso realista del explicacionismo, como "salida" al argumento de la meta-inducción. Sin embargo, Cordero piensa que las críticas que aún puedan quedarle pendientes al selectivismo de Psillos' ${ }^{2}$, como la que señala Diéguez, se pueden resolver señalando una serie de errores que éste comete. El contrargumento de Cordero es concomitante a la respuesta al problema de las entidades inobservables. La demanda de Cordero va en la línea de suspender las etiquetas con las que el resto de estos realistas selectivos apuntan o caracterizan a las teorías. Para Psillos, por ejemplo, una noción como la de "éter luminífero" fue capaz de cambiar en las teorías más avanzadas -en este caso el cambio fue más bien una desaparición- por la sencilla razón de que era un concepto "ocioso", en el sentido de prescindible. A juicio de Cordero, los científicos que empleaban la hipótesis del éter no estaban en disposición de concebir, al menos hasta la llegada de Hertz, un medio distinto para el movimiento de las ondas. En cualquier caso, e incluso aunque hubiese podido ser "ocioso", "[yo] negaría que esta posibilidad ayude al argumento epistemológico a favor del realismo" (Cordero, Sobre el mito 369).

Cordero piensa que el estudio diacrónico -o sea, historiográficode los elementos que pudieron ser centrales en las teorías pasadas encierra un problema sin resolver. Impone a la luz del conocimiento actual aquellos enunciados, modelos, técnicas... que debían ser nucleares para la teoría pretérita. Ahora bien, resulta algo absurdo juzgar desde el presente -conocidos ya los errores en que incurrióqué elementos eran esenciales para la teoría. Quizá haya que tener en cuenta qué pensaban y, sobre todo, qué era lo que podían pensar, los científicos de ese entonces. A veces, no tenían forma de saber qué iba a perpetuarse en las teorías siguientes y, por tanto, qué correspondía al núcleo y qué no.

¿O tal vez sí? ¿Acaso tenían un modo de saberlo? Si respondemos afirmativamente, podríamos empezar a hablar de un enfoque

\footnotetext{
${ }^{2}$ En lo concertinente a las teorías, el realismo de Psillos se podría resumir como sigue: “Psillos, the TP [theoretical posits] $\mathrm{H}$ is essential to success $\mathrm{P}$ and should be considered true or approximately true if and only if: (1) $\mathrm{H}^{\prime}$ and A alone do not lead to P. (2) There is no alternative $\mathrm{H}^{*}$ to $\mathrm{H}$ such that: (a) $\mathrm{H}^{*}$ is consistent with $\mathrm{H}^{\prime}$ and $\mathrm{A}$; (b) $\mathrm{H}^{*}, \mathrm{H}^{\prime}$, and $\mathrm{A}$ lead to $\mathrm{P}$; (c) $\mathrm{H}^{*}$ is not ad hoc or otherwise purposefully concocted to lead to $\mathrm{P}^{\prime \prime}$ (Badino 5).
} 
sincrónico de evaluación. Hablaríamos, además, de una salida al problema que Diéguez achacaba al realismo selectivo, que Cordero cree que afecta particularmente a Psillos. Recordemos: un realista selectivo ha de establecer cuántos elementos centrales se necesitan (y de qué grado de importancia) para poder decir que una teoría ha recogido el testigo de la anterior.

En este punto, Cordero acude a Lakatos para rescatar su examen de las sucesiones teóricas ${ }^{3}$. En la actualidad del examen de una teoría podemos localizar sus partes anquilosadas -Psillos las llamaría "ociosas" - observando de qué modo toleran ser sustituidas y de qué forma dicha sustitución afecta al resto de hipótesis. Cordero interpreta la sugerencia lakatosiana del siguiente modo:

El tratamiento que estoy recomendando de las partes teóricas creíbles se ciñe a las siguientes guías: el gradual estancamiento y éxito independiente de una parte en particular la identificará como "aproximadamente correcta" si es que extirparla o alterarla conduce, consistentemente, a la degeneración empírica del sistema en cuestión y de los sistemas teóricos a los que les es relevante. (Cordero, Sobre el mito 372)

\section{En suma,}

...el aserto realista más contundente que se puede hacer es que, en muchos respectos, los electrones son tales como nos dice la física clásica. De seguro, los desarrollos en la mecánica cuántica desde mediados de la década de 1920 en adelante han enriquecido ampliamente a la representación del electrón, pero las nuevas teorías resultantes han, nuevamente, "mantenido" cuantiosos resultados derivables de la física newtoniana clásica. (Cordero, Sobre el mito 376)

Sería oportuno examinar brevemente, y antes de dar paso al realismo de entidades, un punto que puede poner en entredicho el proyecto

\footnotetext{
${ }^{3}$ Quedaba dividido en dos grandes heurísticas de investigación, una negativa y otra positiva; en palabras del propio Lakatos: "The negative heuristic of the programme forbids us to direct the modus tollens at this "hard core". Instead, we must use our ingenuity to articulate or even invent "auxiliary hypotheses", which form a protective belt around this core, and we must redirect the modus tollens to these. It is this protective belt of auxiliary hypotheses which has to bear the brunt of tests and gets adjusted and re-adjusted, or even completely replaced, to defend the thus-hardened core [...] The positive heuristic consists of a partially articulated set of suggestions or hints on how to change, develop the "refutable variants" of the research programme, how to modify, sophisticate, the "refutable" protective belt" (Lakatos 48-50).
} 
selectivista en lo concerniente a las teorías, esto es, el problema que supone la infradeterminación de las teorías ${ }^{4}$ y la tesis Duhem-Quine. $\mathrm{Si}$ bien parece un dilema al que cualquier selectivista debe hacer frente, lo cierto es que tienden a dejar de lado la cuestión. Cordero sólo lo hace de modo indirecto; aunque rompiendo una lanza a su favor, hemos de decir que el análisis sincrónico, de corte lakatosiano, sí reconoce una cierta vinculación e interdependencia de las partes de las teorías entre sí. El mismo Lakatos tiene muy en cuenta la tesis Duhem-Quine, y a partir de ella sostiene que hay partes de la teoría que no pueden ser refutadas empíricamente ${ }^{5}$.

\section{Realismo de entidades}

El caso del electrón, que es con el que Cordero cerraba su artículo del 2009, es particularmente estudiado por los realistas de entidades. En lo que sigue, trataremos de analizar la propuesta particular de Ian Hacking ("Representing and intervening"), por ser la más representativa dentro de la corriente y seguramente también la que marcó su inicio. Si hubiese que resumirla en pocas palabras, tal vez estas serían las más apropiadas: para Hacking la prueba de que muchas entidades inobservables existen es nuestra habilidad para manipularlas, esto es, para intervenir causalmente en ellas.

\footnotetext{
${ }^{4}$ Quizá haya que recordar este argumento: "The underdetermination of scientific theory by evidence is the simple idea that the evidence available to us at a given time may be insufficient to determine what beliefs we should hold in response to it. In a textbook example, if all I know is that you spent $\$ 10$ on apples and oranges and that apples cost $\$ 1$ while oranges cost $\$ 2$, then I know that you did not buy six oranges, but I do not know whether you bought one orange and eight apples, two oranges and six apples, and so on. A simple scientific example can be found in the rationale behind the sensible methodological adage that "correlation does not imply causation". If watching lots of cartoons causes children to be more violent in their playground behavior, then we should (barring complications) expect to find a correlation between levels of cartoon viewing and violent playground behavior. But that is alsowhat we would expect to find if children who are prone to violence tend to enjoy and seek out cartoons more than other children, or if propensities to violence and increased cartoon viewing are both caused by some third factor (like general parental neglect or excessive consumption of Twinkies). So a high correlation between cartoon viewing and violent playground behavior is evidence that (by itself) simply underdetermines what we should believe about the causal relationship between the two. But it turns out that this simple and familiar predicament only scratches the surface of the various ways in which problems of underdetermination can arise in the course of scientific investigation." (Stanford cap.1).

${ }^{5}$ Como dice Suárez (121): “la idea central de la metodología de los programas de investigación científica es que una división metodológica apropiada entre los supuestos que configuran el núcleo duro, que por razones derivadas de la tesis Duhem-Quine, no pueden ser refutados o confirmados empíricamente, y otros supuestos que configuran el cinturón protector, que sí pueden ser refutados o confirmados por la evidencia empírica, es capaz de modular las conclusiones irracionalistas que se derivan de los paradigmas kuhnianos".
} 
Cuenta este filósofo que su defensa del realismo de entidades y del experimentalismo se debe a una suerte de conversión, de "caída del caballo", que aconteció cuando preguntó a uno de sus profesores en la Universidad de Stanford cómo se conseguía que dos bolitas de niobio, que eran parte de un experimento, tuviesen una carga diferente. Según su maestro, esto se conseguía rociando con positrones para aumentar la carga y con electrones para disminuirla. Fue entonces, cuenta Hacking, cuando cayó en la cuenta de que si era posible rociar algo con electrones, eso debía significar que los electrones existían. No era, pues, una manera de salvar los fenómenos.

Este ejemplo da buena cuenta de la novedad que el realismo de Hacking introduce en torno a los inobservables: el criterio de existencia en base a su manipulabilidad y no tanto a nuestra representación de ellos. Una manipulabilidad que se predica de entidades, pero que no dice mucho de los modelos, de los modelos de fenómenos. Para Hacking hay argumentos que permiten inferir de la manipulabilidad la existencia de entidades. Uno es el que hemos señalado; otro es la recurrencia de los experimentos; y otro es "que no hacemos instrumentos y después inferimos la realidad [...] como cuando ponemos a prueba una hipótesis" (265).

Para Hacking el orden es el inverso. Disponemos de una serie de informaciones de esos inobservables y, después, intervenimos (en ocasiones, esos inobservables sirven para investigar otras entidades). Además, cuando muchos instrumentos coinciden -incluso empleando técnicas y sistemas físicos distintos- en la detección del inobservable, entonces su existencia se afianza, señala Hacking.

De los dos problemas que intenta combatir el realismo selectivo, según Chakravartty, que son el estatuto de los inobservables -sobre el que se acaba de hablar- y la compatibilidad del realismo con la falsedad de las teorías, pareciera que el nombre de "realismo de entidades" ya condenaría desde el principio a esta corriente a dar respuesta solo al primero de los problemas. No obstante, en la obra de Hacking y en algunos otros de sus valedores, hay una preocupación en torno a la verdad de las teorías. Ahora bien, esto no quiere decir que se encuentre aquí otra vindicación realista, sino todo lo contrario. El propio Hacking dedica varias páginas a la cuestión, y aunque comenta que no resulta un problema de su interés, sí afirma que es un error reducir el asunto del progreso y la acumulación del conocimiento al 
mero progreso teórico y a la simple acumulación conceptual (Hacking 56), como pareciera derivarse del argumento de la meta-inducción. Dice Hacking que, incluso cuando esta diversidad se reconoce, se tiende a creer que la acumulación de artefactos, historias naturales y fenómenos apoya de algún modo la verdad de la teoría. Esta relación se funda, a su vez, en la teoría de la verdad como correspondencia, que Hacking rechaza. Incluso aunque existan los electrones, e incluso aunque que tales entidades causen lo que "fenomenológicamente" reconocemos como ciertos eventos del mundo, eso "no es idéntico, ni equivalente, a la inferencia de la verdad de la teoría científica comúnmente aceptada sobre tal ente" (Suárez 200).

Por un lado, estos argumentos separan radicalmente la esfera de la teoría de la de las entidades, en lo que al realismo se refiere. Por otro lado, esta distinción habilita a Hacking para presentar el trabajo experimental como el mejor argumento en favor del realismo. Como dice Hacking "no tiene sentido preguntar por la explicación de nuestro éxito" (Hacking 56. La traducción es mía), y menos aún vincularlo con una teoría. Queda únicamente confiar en que continuará en el futuro. Los experimentos, en suma, tendrían para Hacking una "vida propia", y no verificarían teorías, sino que aportarían información fenoménica del mundo.

Según Valeriano Iranzo, la intuición de Hacking ciertamente casa con nuestra "psicología cotidiana" (62), pero eso no sería suficiente para proponerla como explicación de la práctica científica. Desde Locke, se ha hecho célebre el ejemplo del palo aparentemente deforme cuando se sumerge bajo el agua, como una prueba de la fragilidad de nuestras sensaciones visuales. Ahí Hacking es original en la extrapolación, y a la vez consecuente con la crítica de Locke. No obstante, términos como la "manipulación", en el contexto de la práctica científica, pueden poseer un significado bien distinto del habitual. "Hablar de la manipulación de $\mathrm{x}$ mediante un haz de rayos láser o un acelerador de partículas resulta tan equívoco como hablar de la observación de x a través del microscopio electrónico" (62), pues en ambos casos estarían suponiendo nuestra tecnología, y su existencia sería una atribución bastante indirecta, desde luego no meramente fenoménica, sino mediada por algún tipo de conocimiento teórico. 


\section{Conclusión}

Pese a las críticas esbozadas, no sólo ha quedado demostrado el compromiso realista del selectivismo, sino -y precisamente por eso- el intento de dar respuesta a dos de los más importantes problemas (si no directamente los más importantes) del realismo científico clásico: la existencia de los inobservables y la falsedad de las teorías exitosas. En este sentido, se han examinado las respectivas conclusiones de cada una de las corrientes examinadas: realismo estructural, explicacionismo y realismo de entidades; ello sin perjuicio de que el enfoque de todas las corrientes no se centre por igual en los dos problemas del realismo, y desde luego sin presumir que lo hagan de manera satisfactoria. A la versión de Worrall se la ha objetado con el problema de la cardinalidad de teorías con estructuras matemáticas equivalentes. A Psillos, representante de la primera corriente, se le ha contrapuesto Alberto Cordero, quien depura algunas de sus proclamas, a través de Lakatos; a ambos, a su vez, se les ha mostrado el problema global de la infradeterminación de las teorías. Y en lo que respecta a Hacking y al realismo experimental, la crítica de Valeriano Iranzo permite seguir replanteando su sugerente propuesta.

\section{REFERENCIAS}

Badino, Massimiliano. "How to MakeSelective Realism MoreSelective (and More Realist Too)". The 25th Biennial Meeting of the Philosophy of Science Association. 20/11/2019. http:// philsci-archive.pitt.edu/id/eprint/12523

Chakravartty, Anjan, "Scientific Realism". The Stanford Encyclopedia of Philosophy. Web. 17/04/2020. https://plato.stanford.edu/archives/ sum2017/entries/scientific-realism/

Cordero, Alberto. "Sobre el mito de que el realismo científico ha muerto". Areté. Revista de Filosofía, 21, 2, 2009, pp. 363-379.

---. "Mitos y falsedades sobre el realismo científico". ArtefaCToS. Revista De Estudios Sobre La Ciencia y La Tecnología, 7, 2, 2018, pp. 9-33.

Diéguez, Antonio. "Realismo Científico". Enciclopedia de Filosofía de la Sociedad Española de Filosofía Analítica. Web. 02/02/2020. http://www. sefaweb.es/realismo-cientifico/ 
Ferraris, Maurizio. Manifiesto del nuevo realismo. Ariadna Ediciones, 2012.

Hacking, Ian. Representing and Intervening. Cambridge University Press, 1983.

Iranzo, V. "El dilema del realismo experimental". Episteme NS, 28, 1, 2008, pp. 59-88.

Ladyman, J. Understanding Philosophy of Science. Routledge, 2002.

Lakatos, I. The Methodology of Scientific Research Programmes. Cambridge University Press, 1978.

Laudan, L. "A Confutation of Convergent Realism". Philosophy of Science, 48, 1, 1981, pp. 19-48.

Madrid-Casado, C. “Sr. Realista Estructural, tenemos un problema: la carga ontológica de las matemáticas". Principia, 14, 2, 2010, pp. 201-209.

Psillos, S. Scientific Realism: How Science Tracks Truth. Routledge. 1999.

Rivadulla, A. "Two dogmas of structural realism. A confirmation of a philosophical death foretold". Crítica, 42, 124, 2010, pp. 3- 29.

Stanford, Kyle, "Underdetermination of Scientific Theory". The Stanford Encyclopedia of Philosophy. Web. 30/06/2020. https:// plato.stanford.edu/ archives/win2017/entries/scientific-underdetermination/

Suárez, Mauricio. Filosofía de la ciencia. Historia y práctica. Alianza, 2019.

Van Fraassen, Bass, Chakravartty, Anjan. "What is Scientific Realism?". Spontaneous Generations: A Journal for the History and Philosophy of Science, 9, 1, 2018, pp. 12- 25.

Worrall, John. "Structural realism: The best of both worlds?". Dialectica, 43, 1-2, 1989, pp. 99-124.

\section{Como citar:}

Acosta, Benedicto. "Notas sobre el realismo selectivo y sus respuestas a los problemas del realismo científico". Discusiones filosóficas. Jul. 21 (37), 2020: 57-70. https:// doi.org/10.17151/difil.2020.21.37.4 